\title{
Ocular Neostigmine Drops for Diagnosing Myasthenia Gravis
}

\section{Mustafa A Salih ${ }^{1 *}$, Mohamed A Salih², Ali A Mustafa ${ }^{3}$, Darren T Oystreck ${ }^{4}$, Kamal M Attia ${ }^{5}$, Sara M El-Sadig ${ }^{6}$, Ahlam A Hamed ${ }^{7}$, Waseem M Hajjar $^{8}$ and Thomas M Bosley ${ }^{4}$}

${ }^{1}$ Division of Pediatric Neurology, College of Medicine, King Saud University, Riyadh, Saudi Arabia

2Sinnar Hospital and Kush Eye Center, Omdurman, Sudan

${ }^{3}$ Basic Medical Sciences Department, College of Medicine, King Fahad Medical City, Riyadh, Saudi Arabia

${ }^{4}$ Department of Ophthalmology, College of Medicine, King Saud University, Riyadh, Saudi Arabia

${ }^{5}$ Department of Zoology, College of Science, King Saud University, Riyadh, Saudi Arabia

${ }^{6}$ Department of Medicine, Faculty of Medicine, University of Khartoum, Sudan

${ }^{7}$ Department of Pediatrics and Child Health, Faculty of Medicine, University of Khartoum, Sudan

${ }^{8}$ Department of Surgery, College of Medicine, King Saud University, Riyadh, Saudi Arabia

\begin{abstract}
Introduction: A variety of tests have been devised for the diagnosis of myasthenia gravis (MG). The best known of these (Tensilon test, using intravenous edrophonium chloride) may cause serious complications (bradycardia and syncope) dictating cardiac monitoring during the procedure. Ocular neostigmine drops, a simple procedure, may significantly reduce the risk of diagnostic testing for possible MG.

Method: To investigate its efficacy, the miotic effect of neostigmine was explored using 30 rabbits. One drop of sterile neostigmine solution $(2.5 \mathrm{mg} / \mathrm{ml})$ was instilled into the right eye (RE) of each rabbit using the left eye (LE), which received sterile normal saline, as control. Serial assessments of pupillary size were done. One drop of neostigmine was instilled daily for 7 days to investigate its safety. Six patients (aged $4.5-55 y$, median $=42 y$, mean $=36.4 y$ ) with MG had the same test and were observed for increase of the palpebral fissure height (documented by photography).

Results: At baseline there was no significant difference in the mean [SD] pupillary size of the rabbits between the RE (7 mm [1.07]) and LE (7 mm [1.07]), $p=0.63)$. Significant miotic effect was observed in the RE compared to the LE at 30,60 and 90 minutes (respectively, $4.8 \mathrm{~mm}$ [1.86] vs $7.0 \mathrm{~mm}$ [1.09], $\mathrm{p}=0.0001 ; 4.8 \mathrm{~mm}$ [1.86] vs $7.0 \mathrm{~mm}$ [1.09], $p=0.0001$; and $3.2[0.76]$ vs $7.0[0.0], p=0.013$ ). Administration of one drop of neostigmine daily for 7 days caused no ocular inflammation. All six patients with MG had an observable increase of the palpebral fissure height (documented by photography) of at least $2 \mathrm{~mm}, 30$ minutes after neostigmine instillation. The response was dramatic in the three patients with no prior treatment for MG.
\end{abstract}

Conclusion: Ocular neostigmine drops is a safe, simple and efficient diagnostic test for MG.

Keywords: Myathenia gravis; Diagnosis; Ocular neostigmine drops; Tensilon test; Edrophonium chloride

\section{Introduction}

Myasthenia gravis (MG) is an acquired autoimmune disorder caused by autoantibodies directed against epitopes on or around the acetylcholine receptor (AChR) in the muscle membrane [1]. The condition is characterized clinically by variable ptosis, ocular motility abnormalities, and weakness of voluntary muscles in other parts of the body [1]. Nearly all patients present with extraocular muscles involvement, causing ptosis and diplopia, and in $20-40 \%$ of patients, weakness remains limited to these muscles (ocular MG) [1-3]. The diagnosis may be difficult to make on clinical grounds especially in juvenile cases when weakness is restricted to few muscles such as in ocular MG [4], and a variety of supportive diagnostic tests have been devised [5].

Perhaps the best known of these is the Tensilon test [5-8], using intravenous edrophonium chloride, which is an acetylcholinesterase (AChE) inhibitor with very short action. Unfortunately, edrophonium causes cholinergic side effects that may include increased oral and bronchial secretions, bradycardia, abdominal cramps, sweating, heart block, hypotension, and red, watery, painful eyes. These are often uncomfortable for the patient, and although serious complications of bradycardia and syncope are rare [9] the test is now commonly performed with cardiac monitoring and atropine available as an anticholinergic agent. Other relative contraindications for performance of edrophonium are cardiac dysrhythmias and bronchial asthma. A second quick bedside test is by applying an ice pack locally to a ptotic eyelid for 2-5 minutes and observing for any improvement of ptosis or eye movement deficit [10-12]. The ice pack test is simple, inexpensive, and has a reported sensitivity of $93-97 \%$ and specificity of $97-98 \%$ [13]. This adjunctive diagnostic test is particularly helpful if the edrophonium test is contraindicated or not available. However, the procedure is unlikely to be tolerated by young children [14].

Other supportive tests require laboratory and electrophysiologic facilities. Serologic tests include assessing AChR antibodies which are found in $80 \%$ of adults with MG [15], 70\% of peripubertal juvenile MG but only $50 \%$ of prepubertal MG $[16,17]$. Antibodies to muscle-specific kinase (MuSK) are rare in juvenile MG [18].

Electrodiagnosis of MG includes repetitive nerve stimulation [RNS] (looking for a decrement of greater than $10 \%$ of the fifth compared to the first-evoked compound muscle action potential [CMAP] in

*Corresponding author: Prof. Mustafa A Salih, MD, Department of Pediatrics, College of Medicine, Saudi Arabia, Tel: +966 1 4679728; Fax: +966 14679643 E-mail: mustafa_salih05@yahoo.com

Received February 07, 2013; Accepted March 26, 2013; Published March 30 2013

Citation: Salih MA, Salih MA, Mustafa AA, Oystreck DT, Attia KM, et al (2013) Ocular Neostigmine Drops for Diagnosing Myasthenia Gravis. J Neuro Neurophysiol S11:004. doi:10.4172/2155-9562.S11-004

Copyright: (C) 2013 Salih MA, et al. This is an open-access article distributed unde the terms of the Creative Commons Attribution License, which permits unrestricted use, distribution, and reproduction in any medium, provided the original author and source are credited. 
multiple muscles). However, the test is uncomfortable in children, is artifact prone and can be abnormal in disorders other than MG [14]. The reported sensitivity of RNS ranges between 53\% and 100\%, but it is frequently negative in ocular MG [3]. Single-fiber electromyography (SFEMG) requires a significant degree of cooperation and expertise [18] and is not available in many hospitals. In young children, sedation may be required to facilitate both RNS and stimulated SFEMG [19]. An additional simple, safe, sensitive, and specific diagnostic test for MG is, therefore, needed.

\section{Methods}

This research project investigated the safety and efficacy of using intravenous (IV) neostigmine solution, available in all hospitals where anesthesia is performed, as an ocular drop for diagnostic purposes in the setting of patients with probable or definite autoimmune MG.

The study had two components: 1) experiments using 30 rabbits in which the standard IV neostigmine solution was instilled into one eye (using the other as control) to evaluate effectiveness (i.e., changes in the pupillary size of the experimental eye) and safety (i.e., does not cause significant irritation of the experimental eye or obvious systemic effects). 2) experiments in which the standard IV neostigmine solution was instilled into both eyes of patients with probable or definite symptomatic MG.

Both sets of experiments used the standard neostigmine $(2.5$ $\mathrm{mg} / \mathrm{ml}$ ) solution available in hospitals for anesthetic/surgical use for reversal of neuromuscular block. This solution is buffered and FDAapproved for use in humans. In both sets of experiments, one drop of this solution was used in an eye, limiting substantially the amount of drug that might be absorbed systemically.

\section{Rabbit studies}

Male New Zealand White rabbits (2.5-3.0 kg body weight) were used in all experiments. The animals were kept in a room of controlled room temperature $\left(24.0+1.0^{\circ} \mathrm{C}\right)$ and humidity and a fixed dark light cycle (light on at 7:00 am and off at 19:00 pm).

The sterile neostigmine solution $(2.5 \mathrm{mg} / \mathrm{ml})$ was instilled into the right eye (one drop), while the control eye (left eye) received one drop of sterile saline ( $0.9 \%$ sodium chloride). Assessment of pupillary size was done at 30 minute intervals following instillation of neostigmine. The left eye of each rabbit acted as control for the right one.
The amount of neostigmine methylsulphate was calculated to be $0.1 \mathrm{mg}$ /drop. Therefore, each rabbit's eye was instilled by $0.1 \mathrm{mg}$ of sterile neostigmine methylsulphate. The sterile dropper for instilling neostigmine was used only once and then discarded. To prevent the drop of neostigmine from leaking out of the rabbit's eye, the lower eye lid of the rabbits was held out for at least 2-3 minutes in order to keep the drop inside the eye and allow for better absorption of the drug through the cornea.

The pupil size of both the right and left eyes of each rabbit was measured at baseline in mms by using a plastic ruler. After instilling the neostigmine solution (right eye) or saline (left eye), the pupil size was measured again at 30 minute intervals following drug or saline instillation. One drop of neostimine was instilled daily for 7 days and signs of ocular inflammation were observed.

Statistical analysis: The mean values for the pupil sizes of the right eyes were recorded as well as those of the left eyes, separately. Paired t-test was used for comparison of data among the different groups on SPSS software. A probability value of $<0.05$ was considered to be significant.

\section{Human studies}

Patients with probable or definite MG were asked to sign informed consent approved by the appropriate Institutional Ethics Committees. Ptosis was recorded photographically, and pulse and blood pressure were also recorded prior to beginning the test. One drop of standard IV neostigmine solution $(2.5 \mathrm{mg} / \mathrm{ml})$ was instilled into each symptomatic eye of the patient. Fifteen minutes later, possible changes in ptosis were assessed. Pulse and blood pressure was recorded again and after another 15 minutes. At 30 minutes, the patient was evaluated also for possible changes in ptosis (documented by photography), obvious irritation of either eye and for report of discomfort.

\section{Results}

\section{Rabbit studies}

At baseline (Figure 1) there was no significant difference in the mean [SD] pupillary size of the rabbits between the RE (7 $\mathrm{mm}$ [1.07]) and LE (7 mm [1.07], p=0.63). Significant miotic effect was observed in the RE compared to the LE at 30 and 60 minutes (respectively, 4.8 $\mathrm{mm}$ [1.86] vs $7.0 \mathrm{~mm}$ [1.09], $\mathrm{p}=0.0001$; and $4.8 \mathrm{~mm}$ [1.86] vs $7.0 \mathrm{~mm}$

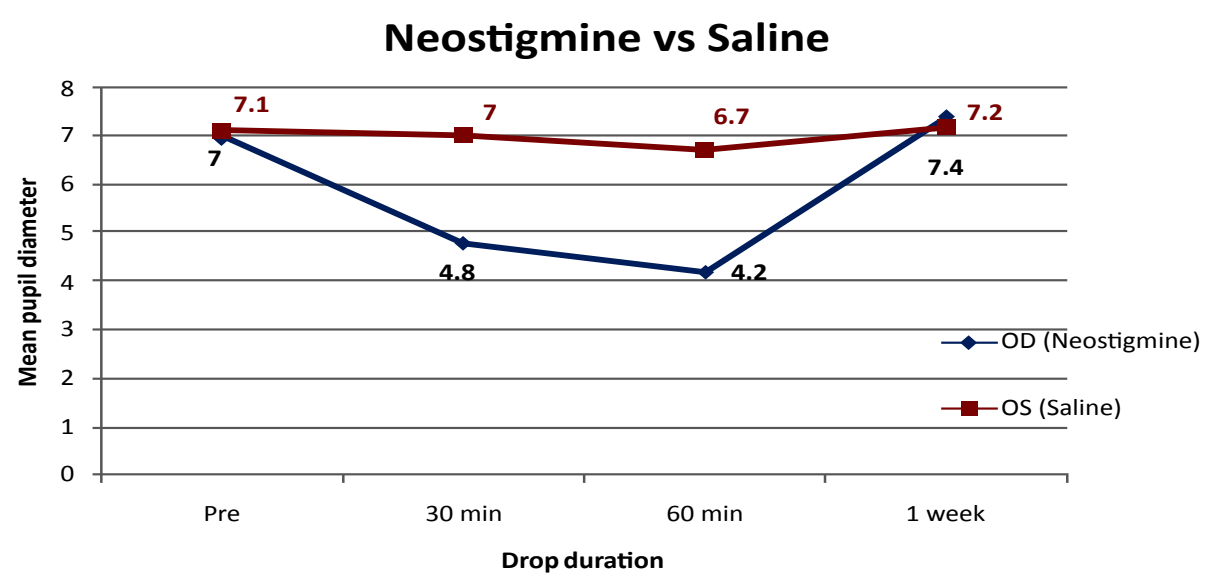

Figure 1: Mean pupil diameter measurements of the right and left eyes of 30 rabbits over time. One drop of neostigmine solution (2.5 mg/ml) was instilled in the right eye, whereas one drop of saline was instilled in the left (control) eye. 
Citation: Salih MA, Salih MA, Mustafa AA, Oystreck DT, Attia KM, et al. (2013) Ocular Neostigmine Drops for Diagnosing Myasthenia Gravis. J Neurol Neurophysiol S11:004. doi:10.4172/2155-9562.S11-004

Page 3 of 4

[1.09], $\mathrm{p}=0.0001)$. This significant miotic effect was also sustained at 90 minutes when comparing the RE to the LE (respectively, 3.2 [0.76] vs 7.0 [0.0], $\mathrm{p}=0.013$ ). Administration of one drop of neostimine daily for 7 days caused no ocular inflammation. Measuring the pupillary size 24 hours after the last drop, revealed no significant difference between the RE and LE (respectively, 7.4 [0.94] vs 7.2 [1.01], p=0.062). A representative photograph of these pupillary changes is shown in Figure 2 .

\section{Patients}

Six patients ( 3 males and 3 females) who presented with ptosis and/or ocular motility disturbance were enrolled in the study (Table 1). Their ages ranged between 4.5 and 55 years (median $=42$ years, mean $=36.4$ years). The duration of their symptoms ranged between 1 and 18 months (median=4 months, mean=5.8 months).Three of them were not suspected before or investigated for MG, and hence received no treatment (Patients 1, 2 and 5). The other 3 (Patients 3, 4 and 6) were seropositive for AChR-antibodies, and one (Patient 6) had, as well, significant decrement $(>10 \%)$ on repetitive nerve stimulation (RNS). Patient 3 (Table 1) had prednisone and pyridostigmine for 3 months, another (Patients 4) had prednisone for 2 months, and a third (Patient 6) had pyridostigmine for one year. Patient 3 received $30 \mathrm{mg}$ of pyridostigmine 2 hours prior to the test whereas Patient 6 had $60 \mathrm{mg}$ of pyridostigmine 8 hours earlier. The 6 patients manifested clinically with ocular MG, with no limb, axial muscles, oropharyngeal or respiratory muscle involvement [5].

All 6 patients with MG had an observable increase of the palpebral fissure height (documented by photography) of at least $2 \mathrm{~mm}, 30$ minutes after neostigmine instillation (Figure 3 ). The response was dramatic in the 3 patients with no prior treatment for MG (Patients 1,2 and 5).

\section{Discussion}

Population based epidemiological studies in MG reported an incidence rate ranging from 1.7 to 21.3 cases per million person per year and prevalence rate ranging from 15 to 179 per million [20]. In both sexes, the incidence rate was reported to increase with age, peaking between 60 and 80 years, being more predominant in the older age group [20]. The proportion of childhood-onset ( $<15$ years) MG appears to be relatively high in Asians [21]. Also in a series from Saudi Arabia [22], children ( $\leq 16$ years) accounted for $20 \%$ of 150 patients who had thymectomy for the treatment of MG. The incidence of juvenile patients who present with ocular MG has been reported to

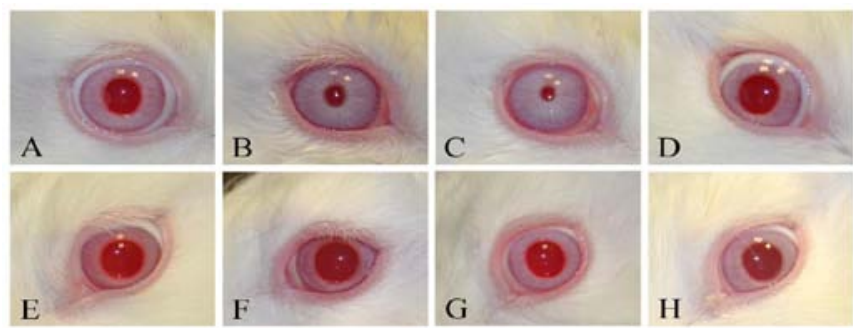

Figure 2: Pupillary response to instillation of neostigmine (right eye) and saline (left eye). Images A-D=right eye; Images $\mathrm{E}-\mathrm{H}=$ left eye. A=Pre neostigmine $(6.0 \mathrm{~mm}) ; \mathrm{B}=30$ mins post neostigmine $(2.0 \mathrm{~mm}) ; \mathrm{C}=60$ mins post neostigmine $(1.5 \mathrm{~mm}) ; \mathrm{D}=1$ week after daily instillation of 1 drop of neostigmine, last drop 24 hours previously $(6.0 \mathrm{~mm})$; $E=$ Pre saline $(7.0 \mathrm{~mm}) ; \mathrm{F}=30 \mathrm{mins}$ post saline drop (7.5mm); $\mathrm{G}=60 \mathrm{mins}$ post saline drop $(7.0 \mathrm{~mm}) ; \mathrm{H}=1$ week after daily instillation of 1 drop of saline, last drop 24 hours previously $(7.0 \mathrm{~mm})$.

\begin{tabular}{|c|c|c|c|c|c|}
\hline $\begin{array}{c}\text { Patient } \\
\text { (P) No. }\end{array}$ & Gender & Age & $\begin{array}{c}\text { Duration of } \\
\text { symptoms }\end{array}$ & Diagnostic test (s) & $\begin{array}{c}\text { Treatment prior to } \\
\text { ocular neostigmine } \\
\text { (duration) }\end{array}$ \\
\hline P1 & M & $55 y$ & $3 \mathrm{mo}$ & ND & None \\
\hline P2 & M & $37 y$ & $1 \mathrm{mo}$ & ND & None \\
\hline P3 & F & $41 / 2 y$ & $5 \mathrm{mo}$ & $\begin{array}{c}\text { Positive AChR } \\
\text { antibody }\end{array}$ & $\begin{array}{c}\text { Prednisolone and } \\
\text { pyridostigmine (3 mo) }\end{array}$ \\
\hline P4 & F & $48 y$ & $5 \mathrm{mo}$ & $\begin{array}{c}\text { Positive AChR } \\
\text { antibody }\end{array}$ & Prednisone (2 mo) \\
\hline P5 & M & $27 y$ & $3 y$ & ND & None \\
\hline P6 & F & $47 y$ & $11 / 2 y$ & $\begin{array}{l}\text { Positive AChR } \\
\text { antibody. Significant } \\
\text { decrement on RNS }\end{array}$ & Pyridostigmine (1y) \\
\hline
\end{tabular}

Abbreviations: AChR: Acetylcholine Receptor; F: Female; M: Male; mo: month ND: Not Done; RNS: Repetitive Nerve Stimulation; y: years.

Table 1: Clinical features of 6 patients with ocular myasthenia gravis.
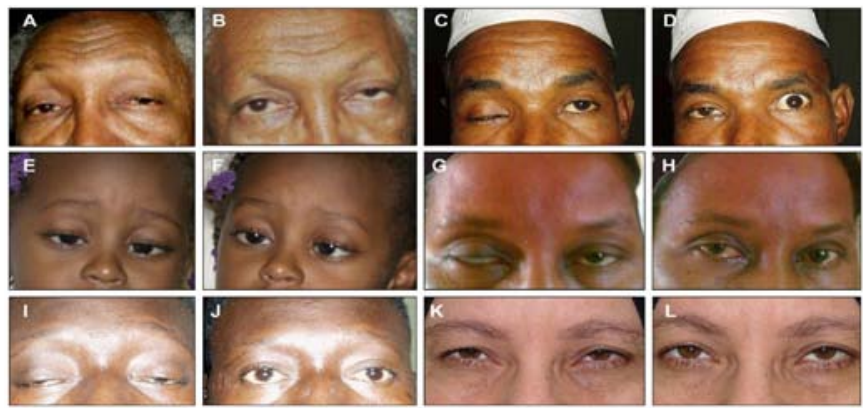

Figure 3: Ocular neostigmine test in 6 patients with myasthenia gravis (MG). $(A, C, E, G, I, K)$ baseline ptosis in Patients 1-6, respectively. (B, D, F, H $\mathrm{J}, \mathrm{L})$ Observable increase of the palpebral fissure 30 minutes after instillation of neostigmine drops. The response was striking in Patients 1, 2 and 5 (respectively, B, D and J) who had not received treatment for MG.

range from $27 \%$ to $93 \%$, with substantially high incidence of ocular presentation in Asian children [18]. A trend towards developing ocular MG rather than generalized MG was reported from South Africa in children of African ancestory [23], with an age peak between 2 and 4 years [24]. It's noteworthy that under-recognition of MG is likely in Developing Countries where access to specialist services is poor [25].

Although the clinical course of MG is variable, spontaneous remission is still uncommon and the time of diagnosis from onset $(<1$ year) and intervention was reported to be the most robust prognostic factor for remission [26]. Nevertheless, clinical diagnosis may be difficult especially when weakness is restricted to few muscles such as ocular MG.

The two bedside tests with high sensitivity and specificity include the ice pack and edrophonium tests. Nevertheless, application of ice over the eyelid for 2-5 minutes is unlikely to be tolerated by children (personal observation). Edrophonium test, which requires IV infusion of the AChE inhibitor edrophonium chloride, has a reported sensitivity of $71.5-95 \%$ and specificity of $97 \%$ [2,27]. The sensitivity of the test is likely to belower in the busy clinical practice [2]. Moreover, false-positive tests (which have been reported with Guillain-Barre syndrome, brain stem lesions and compressive cranial neuropathies) might be higher in these settings $[2,27]$. Due to the potential serious cholinergic effects, edrophonium test in children should be conducted in a monitored setting with availability of atropine and staff trained in cardiac resuscitation $[1,18,19]$. Some patients with congenital myasthenic syndromes may deteriorate when exposed to an AChE inhibitor such as COLQ and MuSK mutations [28,29]. Also edrophonium is not 
readily available in many hospitals of the Developing Countries. This is what prompted one of the of Authors (Mo A S) during his work in a district hospital in Sudan, to pioneer the idea of ocular neostigmine as a diagnostic test for MG. The neostigmine preparation $(2.5 \mathrm{mg} / \mathrm{ml})$ which has been used as ocular drops in the present study is available as IV solution $(2.5 \mathrm{mg} / \mathrm{ml})$ in all hospitals where anesthesia is performed.

The rabbits were chosen to test for the efficacy and safety of neostigmine eye drops since they are established experimental model for research on $M G$ [30,31]. The rabbit experiment was included to show that the concentration of the drug is in the correct range, and that the drug does not cause irritation. Significant miotic effect of ocular neostigmine could be demonstrated at 30 and 60 minutes following its instillation (Figures 1 and 2). This significant miotic effect was also evident at 90 minutes. No ocular inflammation was observed even after administration of neostigmine daily for 7 days.

The application of neostigmine drops for 3 patients, who received no prior treatment for MG because the disease has not been suspected before, showed dramatic response (Figure 3). It has been reported that MG is likely to remain restricted to the ocular muscles if the patient had restricted ocular manifestation of the disease for more than 2 years, similar to what has been observed in Patient 5 (Table 1) who had ocular MG for duration of 3 years [2]. The other patients (Table 1), who were all seropositive for AChR antibody, had prior treatment for MG (prednisone and/or pyridostigmine) and the disease has also been confirmed by significant decrement on RNS in a 47-year-old female (Patient 6, Table 1) who had the longest duration of therapy (pyridostigmine for one year). Although the response to neostigmine drops was less dramatic than in the first 3 patients who received no treatment for MG, an observable increase of the palpebral fissure height of at least $2 \mathrm{~mm}$ was observed 30 minutes after neostigmine instillation (Figure 3). This sub-group included a $4 \frac{1}{2}$-year-old girl in whom edrophonium test is expected to be met with technical difficulties, as well as safety reservations. No ocular or systemic side effects were observed in any of the 6 patients.

\section{Conclusions}

Based on the present experimental and clinical research, ocular neostigmine drops proved to be an alternative safe, simple and efficient diagnostic test for MG. It may fulfill the need in many regions around the world where the other standard tests for MG are either unavailable or technically not feasible.

\section{Acknowledgements}

The authors wish to thank Jamal B. Elhardallo for the excellent technical help. Thanks are also due to Vir Salvador for medical illustration and Loida M. Sese for secretarial assistance.

\section{References}

1. Salih MA (2012) Neuromuscular transmission disorders. In: Elzouki AY, (eds) Textbook of Clinical Pediatrics. Springer-Verlag, Berlin Heidelberg, New York.

2. Luchanok U, Kaminski HJ (2008) Ocular myasthenia: diagnostic and treatment recommendations and the evidence base. Curr Opin Neurol 21: 8-15.

3. Spillane J, Beeson DJ, Kullmann DM (2010) Myasthenia and related disorders of the neuromuscular junction. J Neurol Neurosurg Psychiatry 81: 850-857.

4. Afifi AK, Bell WE (1993) Tests for juvenile myasthenia gravis: comparative diagnostic yield and prediction of outcome. J Child Neurol 8: 403-411.

5. Mittal MK, Barohn RJ, Pasnoor M, McVey A, Herbelin L, et al. (2011) Ocular myasthenia gravis in an academic neuro-ophthalmology clinic: clinical features and therapeutic response. J Clin Neuromuscul Dis 13: 46-52.

6. Meriggioli M (2009) Myasthenia gravis: immunopathogenesis, diagnosis, and management. Continuum 15:35-62.
7. Rowin J (2009) Approach to the patient with suspected myasthenia gravis or ALS: a clinician's guide. Continuum 15:13-34.

8. Meriggioli MN, Sanders DB (2009) Autoimmune myasthenia gravis: emerging clinical and biological heterogeneity. Lancet Neurol 8: 475-490.

9. Okun MS, Charriez CM, Bhatti MT, Watson RT, Swift T (2001) Tensilon and the diagnosis of myasthenia gravis: are we using the Tensilon test too much? Neurologist 7: 295-299.

10. Sethi KD, Rivner MH, Swift TR (1987) Ice pack test for myasthenia gravis. Neurology 37: 1383-1385.

11. Golnik KC, Pena R, Lee AG, Eggenberger ER (1999) An ice test for the diagnosis of myasthenia gravis. Ophthalmology 106: 1282-1286.

12. Ellis FD, Hoyt CS, Ellis FJ, Jeffery AR, Sondhi N (2000) Extraocular muscle responses to orbital cooling (ice test) for ocular myasthenia gravis diagnosis. J AAPOS 4: 271-281.

13. Benatar M (2006) A systematic review of diagnostic studies in myasthenia gravis. Neuromuscul Disord 16: 459-467.

14. Salih MA, Oystreck DT, Al-Faky YH, Kabiraj M, Omer Ml, et al. (2011) Congenital myasthenic syndrome due to homozygous CHRNE mutations: report of patients in Arabia. J Neuroophthalmol 31: 42-47.

15. Drachman DB (1994) Myasthenia gravis. N Engl J Med 330: 1797-1810

16. Andrews PI, Massey JM, Howard JF Jr, Sanders DB (1994) Race, sex, and puberty influence onset, severity, and outcome in juvenile myasthenia gravis. Neurology 44: 1208-1214.

17. Andrews PI, Massey JM, Sanders DB (1993) Acetylcholine receptor antibodies in juvenile myasthenia gravis. Neurology 43: 977-982.

18. Chiang LM, Darras BT, Kang PB (2009) Juvenile myasthenia gravis. Muscle Nerve 39: 423-431.

19. McMillan HJ, Darras BT, Kang PB (2011) Autoimmune neuromuscular disorders in childhood. Curr Treat Options Neurol 13: 590-607.

20. Carr AS, Cardwell CR, McCarron PO, McConville J (2010) A systematic review of population based epidemiological studies in Myasthenia Gravis. BMC Neurol 10: 46.

21. Yu YL, Hawkins BR, Ip MS, Wong V, Woo E (1992) Myasthenia gravis in Hong Kong Chinese. 1. Epidemiology and adult disease. Acta Neurol Scand 86: 113119

22. Essa M, El-Medany Y, Hajjar W, Hariri Z, Al-Mulhim F, et al. (2003) Maximal thymectomy in children with myasthenia gravis. Eur J Cardiothorac Surg 24 187-189.

23. Heckmann JM, Hansen P, Van Toorn R, Lubbe E, Janse van Rensburg E, et al (2012) The characteristics of juvenile myasthenia gravis among South Africans. S Afr Med J 102: 532-536.

24. Evoli A (2010) Acquired myasthenia gravis in childhood. Curr Opin Neurol 23 536-540.

25. Bateman KJ, Schinkel M, Little F, Liebenberg L, Vincent A, et al. (2007) Incidence of seropositive myasthenia gravis in Cape Town and South Africa. S Afr Med J 97: 959-962

26. Mao ZF, Mo XA, Qin C, Lai YR, Olde Hartman TC (2010) Course and prognosis of myasthenia gravis: a systematic review. Eur J Neurol 17: 913-921.

27. Pascuzzi RM (2003) The edrophonium test. Semin Neurol 23: 83-88.

28. Mihaylova V, Müller JS, Vilchez JJ, Salih MA, Kabiraj MM, et al. (2008) Clinical and molecular genetic findings in COLQ-mutant congenital myasthenic syndromes. Brain 131: 747-759.

29. Mihaylova V, Salih MA, Mukhtar MM, Abuzeid HA, El-Sadig SM, et al. (2009) Refinement of the clinical phenotype in musk-related congenital myasthenic syndromes. Neurology 73: 1926-1928.

30. Ueno S, Kang J, Takeuchi H, Takahashi M, Tarui S (1980) Specificities of antibody to acetylcholine receptor in rabbits with experimental myasthenia gravis. Clin Exp Immunol 41: 13-18.

31. Ueno S, Wada K, Takahashi M, Tarui S (1980) Acetylcholine receptor in rabbit thymus: antigenic similarity between acetylcholine receptors of muscle and thymus. Clin Exp Immunol 42: 463-469. 